\title{
EMOTION RECOGNITION BASED ON EEG FEATURES WITH VARIOUS BRAIN REGIONS
}

\author{
Kalyani P. Wagh, \\ Research Scholar, Sathyabama Institute of Science \& Technology, Chennai, Tamilnadu, India \\ kalyaniwagh13@gmail.com \\ K.Vasanth \\ Professor, Vidya Jyothi Institute of Technology, Hyderabad, Telangana, India \\ vasanthecek@gmail.com
}

Sagar Shinde

Associate Professor, PCET's NMVP's Nutan College of Engineering \& research, Pune, Maharashtra, India

Abstract: sagar.shinde5736@gmail.com

Currently researchers have shown immeasurable awareness in Brain Computer Interface (BCI) systems, which enable any user to exchange intelligence and knowledge with surrounding and control instruments by using brain signals; concept is identified as Affective Computing. In this work we are using the SEED database, which is publically available to classify three emotions Positive, Negative and Neutral. Five electrode pairs from various brain regions like Prefrontal, Frontal, Temporal, Parietal and Occipital are selected for this work based on previous research. Diverse time domain and time frequency domain features are extracted from EEG signals. Wavelet Transform (WT) is used to extract a variety of time frequency domain features. Daubechies wavelet function (db6) with 6 levels of decomposition is used to split EEG signals into various frequency bands $(\delta, \theta, \alpha, \beta$ and $\gamma)$. SVM and k-NN algorithms are used as classifiers to estimate classification performance. Hypothetical results illustrate an average classification accuracy of 62.4\% for classifying three emotions. Gamma and Beta, the higher frequency bands perform well in emotion recognition.

Keywords: EEG, Emotion recognition, Wavelet Transform, SVM, KNN

\section{Introduction}

Human emotion is a psychological and incredibly complicated state of human being having correlation with mental activity. It can be intertwined with things like behavior, feelings, thoughts, reactions to internal or external stimuli, and so on. In today's technologically advanced world, emotion recognition is a critical issue. Researchers are working on various technologies and methodologies such as Artificial Intelligence, Machine Learning, Logistic Regression, Extreme Machine Learning, Deep Learning, Fast Fourier Transform, Neural Network, Principal Component Analysis (PCA), and Power Spectral Deficiency (PSD), and so on to improve HumanComputer Interaction (HCI). It is necessary because people are so inextricably linked to various types of machines in order to solve daily problems. Medical diagnosis and analysis, e-learning, home and city automation, music therapy, AI-based conversations, social media, video gaming, online shopping, recommendation systems, safe driving, military, and so on are just a few examples. For such applications it is critical task in real time to make machine adaptable of humans' emotional state and user friendly.

Human emotions can be recognized by various techniques like facial expressions, speech tone and gesture appearance. But often these emotions are not sufficient to detect internal true emotion since many times these external emotions are fake. This is one kind of approach to identify emotion. Another approach is, as emotions are biological status, associated with the nervous system. These conditions are brought on by neurophysiological changes generally related with opinion, feelings, and behavioral responses. The Brain is most important and valuable part of the human body and the resource of internal true emotion. A several methods and approaches have been suggested by various researchers to disclose relation between emotion and EEG signal [Md. Asadur et.al.(2019), Kalyani et.al.(2020)]. The Researchers used various physical parameters like Electrocardiogram (ECG), Electromyogram (EMG), Heart rate and Skin conductive resistance (SCR) for recognizing emotions. Some investigators used speech and facial expression for emotion classification.

The Electroencephalogram signals are used to detect human emotions by placing electrodes on the human skull [Faust et.al.(2018)]. Many researchers have done a lot of research in this area, however recognizing an 
emotion for machine is very challenging task [Thipathi et.al. (2018)]. In previous study researchers applied time frequency distribution and various spectral analysis techniques like discrete wavelet transform (DWT) [Anagnostopoulos et.al.(2015)] and Fourier transform (FT) [Mert et.al.(2018)] for identification of emotion. As EEG signal is non stationary in nature, its frequency components change with time. As we know that only time components or frequency components are not enough to identify emotion. So to attain occupied knowledge of an EEG signal in temporal and spatial domain one are using continuous wavelet transform (CWT). It is challenging task to recognize different emotions of different subjects because of non linear nature of EEG signal. Thus selection of channels and various features is critical task in identifying human emotions correctly [Sadiq et.al.(2019), Kevric et al.(2017)] used wavelet packet decomposition method to decompose EEG signal. They used same method for denoising the signal and achieve classification accuracy of 92.8\%. [Gupta et al. (2019)] proposes wavelet based feature extraction techniques for SEED database. Authors used flexible analytical wavelet transform, channel specific technique, which selects channel using machine learning techniques. They attain accuracy of 83.3\%. [Zangeneh et al.(2018)] gives feature selection method with frontal and occipital electrodes for classification and got accuracy of $90 \%$. Selection of appropriate features is helpful to maximize performance of classifier. Thus it reduces the dimensionality of feature set. There are many techniques which can be applied to prevent feature selection problems. First is filtering approach based on ranking technique, which selects functions based on scores tasks based on their relevance and defining thresholds for filtering out irrelevant features.[Mert et al.(2018)] classify two dimensional emotional states using SVM and ANN classifiers by differential entropy on various rhythms is used as a feature. According to the authors [Zhang et.al.(2019), Duan et.al(2013)] beta and Gamma band are most effective for emotion recognition. Researchers study various linear and non linear time frequency features using neural network for classification. [Thammasan et. al.(2016)] explains how selection of specific channels is important for emotion recognition. Author works on 32 channels and ten specific channels ( $\mathrm{F} 3, \mathrm{~F} 4, \mathrm{FP} 1, \mathrm{FP} 2, \mathrm{P} 3, \mathrm{P} 4, \mathrm{~T} 7, \mathrm{~T} 8,01$ and O2). The accuracy of ten channels is better than 32 channels.[Jie et.al. (2014)] author uses entropy of samples as a feature to classify emotions. Author selects front lobe electrodes F3, $\mathrm{CP} 5, \mathrm{FP} 2, \mathrm{FZ}$ and $\mathrm{FC} 2$ for their research. Few works have investigates the spatial domain individuality of multi channel EEG and it may also contain useful information.

In previous study, researchers used selective number of channels to recognize human emotions. This increases the computation speed but decreases the accuracy rate. [Wagh et.al.(2020)] uses different pairs of channel (12 channels, 9 channels, 6 channels and 4 channels) for emotion classification using various wavelet coefficients and entropy as features. SVM and KNN classifiers are used for classification. Despite many of BCI's techniques for capturing EEG signals for emotion classification are there. However there is still scope for improvement in extracting features including accuracy.

\section{Experiment Design}

Various phases like collection of EEG data, pre-processing of EEG signal, feature extraction and their importance, feature reduction and classification are included in proposed system.

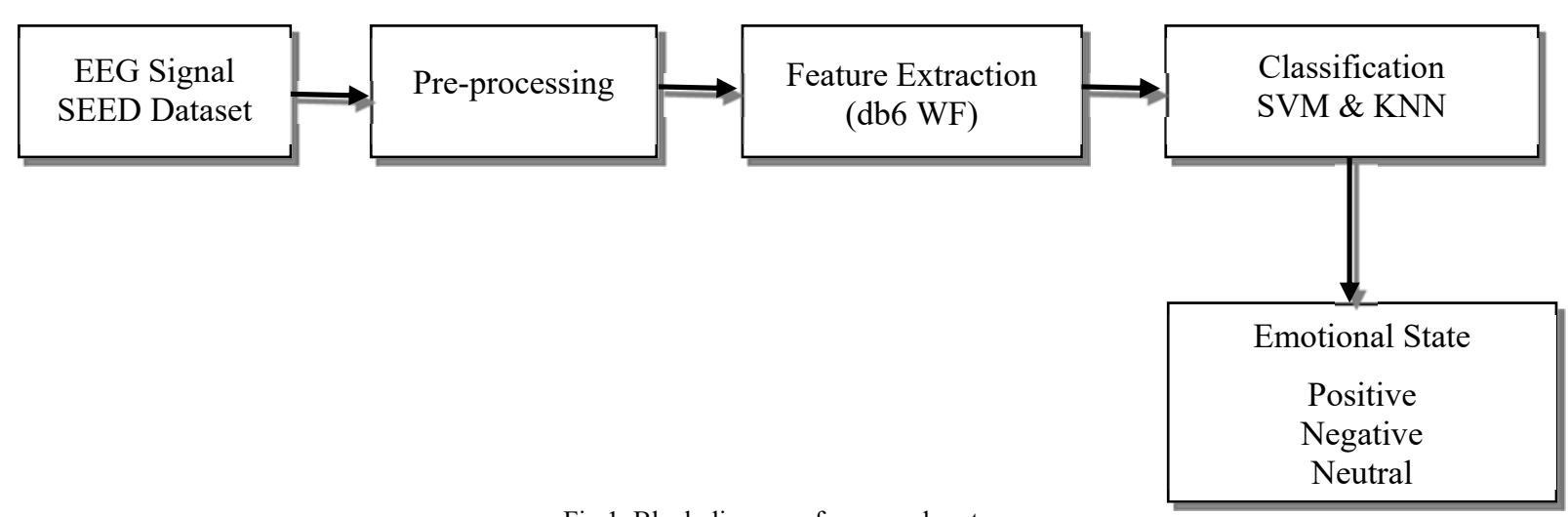

Fig.1. Block diagram of proposed system

\subsection{SEED Database}

SJTU Emotion EEG Dataset (SEED) is a collection of EEG dataset provided by the BCMI laboratory. The dataset is for emotion analysis and it is freely available. Total 15 Chinese film clips with 4 minutes duration (Positive, Neutral and Negative emotions) were chosen as stimuli for this experiment. Fifteen Chinese subjects (7 males and 8 females) contributed in the experiments. There are totally 15 trials for each experiment. Each subject performed the experiment three times with an interval of about one week. The data was down sampled to $200 \mathrm{~Hz}$. Data was preprocessed using bandpass filter from $0-75 \mathrm{~Hz}$. 


\subsection{Channel Selection}

Asymmetries of left and right lobe of brain are generally used in emotion detection. According to the previous research, the left hemisphere of brain processes positive emotions and right hemisphere of brain processes negative emotions. Thus left lobe of brain is concerned in high values of valence like positive, happy emotions and right lobe of brain is concerned in lower valence like angry, sad. By considering pervious work, we select five electrode pairs Prefrontal (FP1-FP2), Frontal (F3-F4), Temporal (T3-T4), Parietal (P7-P8) and Occipital (O1-O2) out of 62 electrodes.

\subsection{Feature Extraction}

Extracting meaningful information from preprocessed EEG signal is Feature Extraction. A quantity that represents uniqueness between classes is a feature. It is a numerical value characterizing a data or providing some information about the data.

\subsubsection{Time domain features}

In this paper, we are using five different kinds of time domain features.

(1) Standard Deviation of raw EEG signal -

$$
\sigma_{\mathrm{X}}=\left(\frac{1}{\mathrm{~N}-1} \sum_{\mathrm{n}=1}^{\mathrm{N}}(\mathrm{X}(\mathrm{n})-\mu \mathrm{x})^{2}\right)^{1 / 2}
$$

where $\mathrm{X}(\mathrm{n})$ represents the value of $n^{\text {th }}$ sample of EEG signal, $\mathrm{n}=1,2, \ldots . \mathrm{N}$

(2) The Mean of raw signal -

$$
\mu \mathrm{x}=\frac{1}{\mathrm{~N}} \sum_{\mathrm{n}=1}^{\mathrm{N}} \mathrm{X}(\mathrm{n})
$$

(3) Skewness - The lack of symmetry or the asymmetry of an EEG signal can be measure with this parameter. A positive value of skewness specifies that an EEG signal is scattered more to the left of the mean value. A Negative value shows that the EEG signal is dispersed more to the right of the mean.

$$
\gamma=\frac{\mathrm{E}\left[(\mathrm{x}(\mathrm{n})-\mu)^{3}\right]}{\sigma^{3}}
$$

(4) Kurtosis - It is a statistical parameter used to measures the complexity of an EEG signal.

$$
\text { Kurtosis }=\frac{E\left[(x(n)-\sigma)^{4}\right]}{\left.\left[E[x(n)-\sigma)^{2}\right]\right]^{2}}
$$

where $E$ is the expected value estimator of the signal $x(n)$

(5) Hjorth Parameters - It is time domain feature used to analyze EEG signal. Activity, Mobility and Complexity are three parameters we can measure.

$$
\begin{gathered}
\text { Activity }=\sum_{t=1}^{T} \frac{(X(t)-\mu)^{2}}{T} \\
\text { Mobility }=\sqrt{\frac{\operatorname{var}\left(X^{\prime}(t)\right)}{\operatorname{var}(X(t))}} \\
\text { Complexity }=\frac{\operatorname{Mobility}\left(X^{\prime}(t)\right)}{\operatorname{Mobility}(X(t))}
\end{gathered}
$$

Here activity is nothing but signal power, Mobility is mean frequency and Complexity is change in frequency.

\subsubsection{Frequency domain features}

Various mathematical tools like Fourier Transform, Fast Fourier Transform, and Short Time Fourier Transform are available for EEG feature extraction. In this work we use Wavelet Transform to extract various time frequency domain features as this method is suitable for non stationary signal like EEG. Discrete Wavelet Transform is used to decomposition EEG signal in various frequency bands. We are using Daubechies 'db6' type wavelet transform with 6 decomposition levels to get various frequency bands like $\delta, \theta, \alpha, \beta$ and $\gamma$. DWT uses a technique known as Multi Resolution Analysis (MRA) in which EEG signal is break up into various coefficients called as 
approximation (CA) and detailed (CD) coefficients. Afterward approximation signal once again decomposed into new $\mathrm{CA}$ and $\mathrm{CD}$ variables.

$$
X w t(\tau, s)=\frac{1}{\sqrt{|s|}} \int_{-\infty}^{+\infty} x(t) \varphi *\left(\frac{t-\tau}{s}\right) d t
$$

where $x(t)$ is raw EEG signal, sis scale factor and $\varphi$ is mother wavelet.

\begin{tabular}{cccc}
\hline Decomposition Levels & $\begin{array}{c}\text { Frequency Bands } \\
\mathbf{( H z )}\end{array}$ & Decomposed Signals & EEG Bands \\
\hline 1 & $100-200$ & D1 & Noise \\
2 & $50-100$ & D2 & Higher Gamma \\
3 & $25-50$ & D3 & Lower Gamma \\
4 & $12-25$ & D4 & Beta \\
5 & $6-12$ & D5 & Alpha \\
6 & $3-6$ & D6 & Theta \\
6 & $0-3$ & A6 & Delta \\
\hline
\end{tabular}

Table 1. Decomposition of EEG signal into five frequency bands and noise
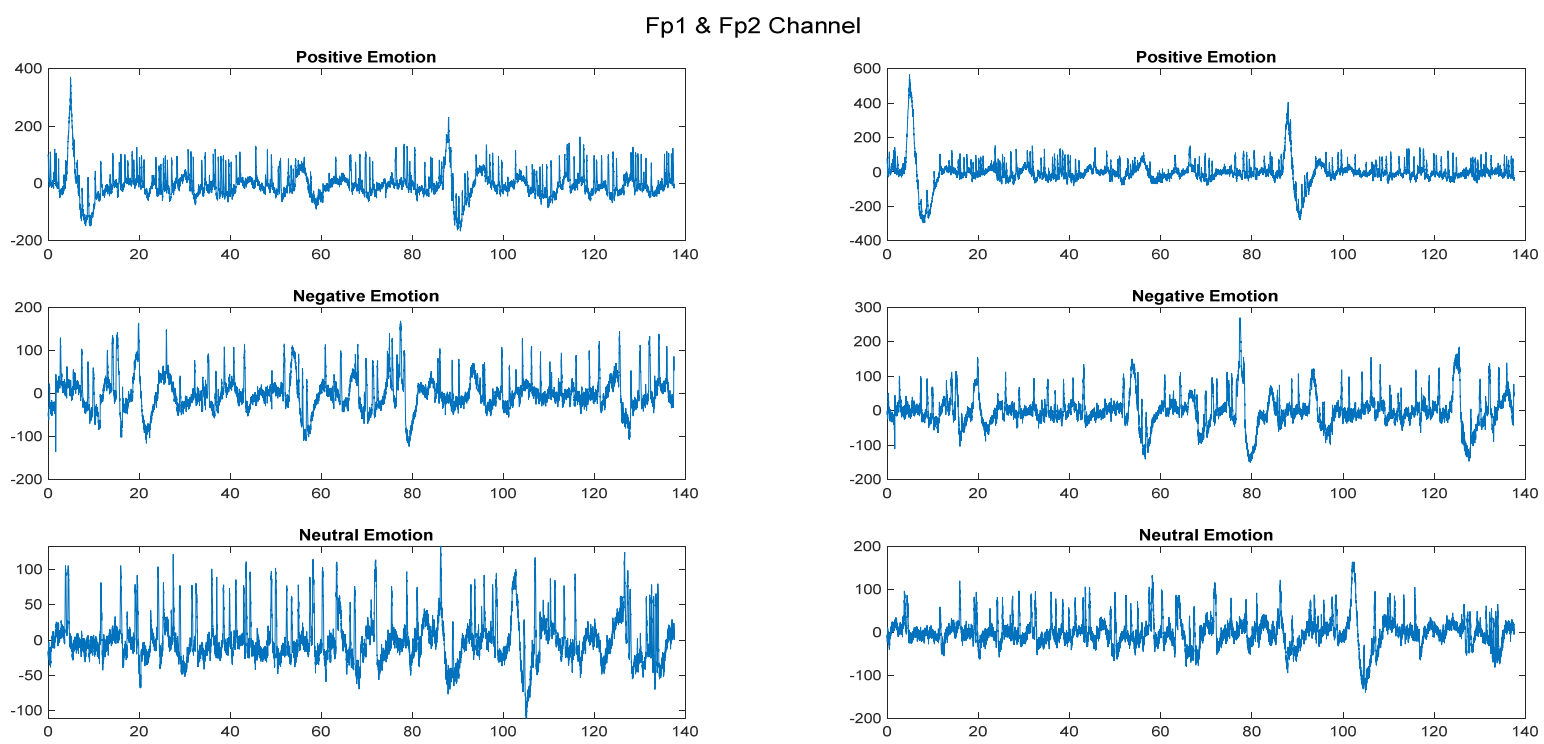

Fig. 2: Time domain EEG signal from channel FP1 \& FP2 for various emotions 


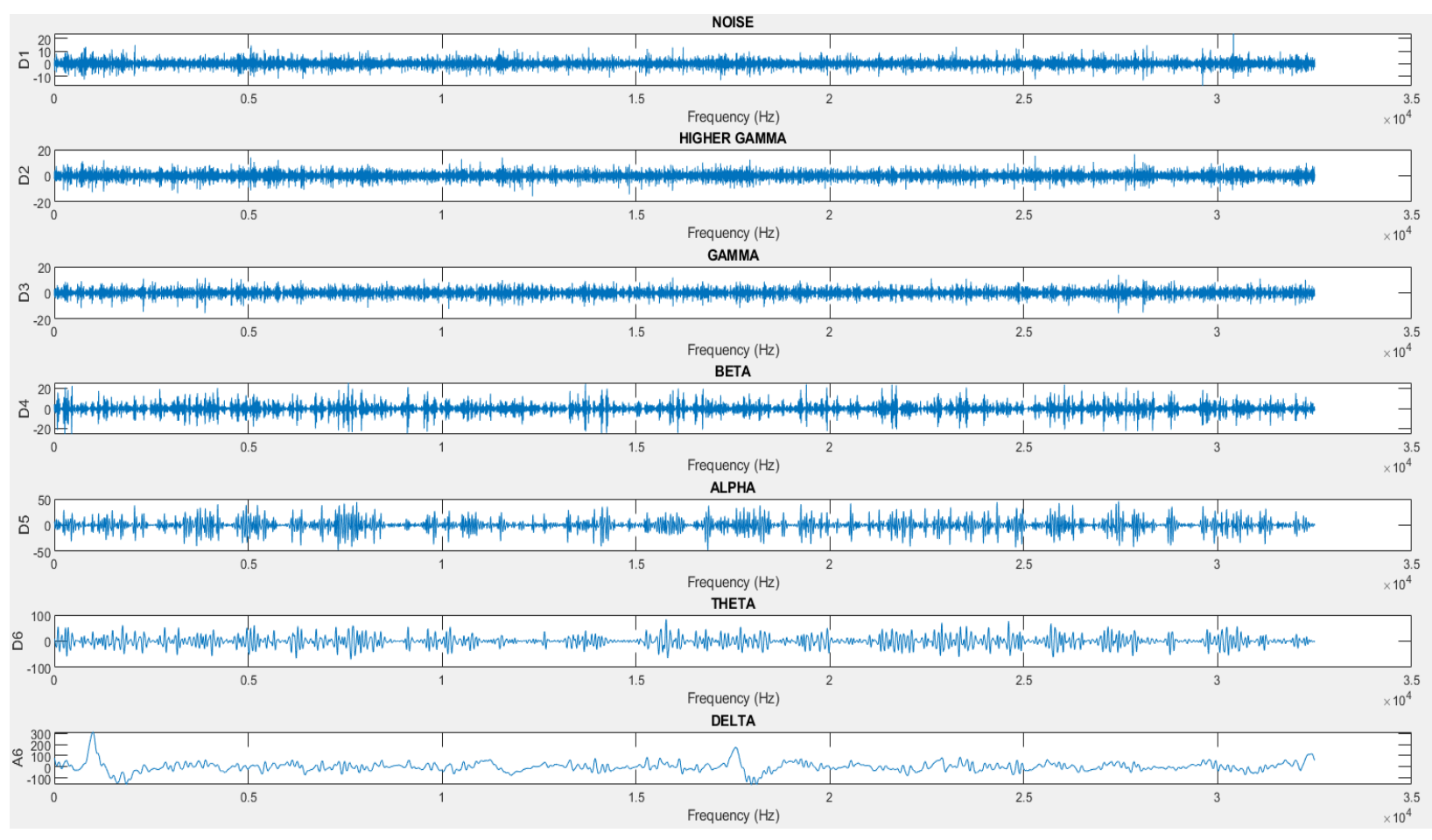

Fig. 3. Decomposition of EEG signal in various frequency bands using db6 with 6 levels of signal decomposition

After getting various frequency bands we have calculated Power Spectral Density using pwelch method with Hamming window of size 200 samples. Then we have calculated various features like Power, Standard Deviation, Variance of various frequency bands delta, theta, alpha, beta and gamma. Thus we have calculated five time domain features and five frequency domain features for our work.

\subsection{Emotion Classification}

In this work, we analytically evaluate classification assessment of support vector machine (SVM), K nearest neighbor (KNN) for emotion identification. $70 \%$ of data is used for training the model and $30 \%$ data is used for testing purpose.

Support Vector Machine (SVM) is a supervised data classification algorithm with good scalability. By making predictive analysis it allocates new data elements to one of labeled group. In the SVM algorithm, each data item will be pointed as $n$-dimensional space, where $\mathrm{n}$ is number of features. The value of each feature being the value of a particular coordinate. Then, classification will be done by finding the hyper-plane which discriminates the two classes. The points near to the hyper-plane called as support vectors.

K-nearest neighbors (KNN) algorithm is non parametric estimation algorithm which is simple, easy-toimplement. It can be used to solve classification and regression problems. It considers the relationship between new data and available data. Then it puts new data into the group which is similar to available group. K-NN is a non-parametric algorithm, means it does not make any estimates on original data.

\section{Experiment Results}

In this work all three emotions (Positive, Negative and Neutral) are analyzed using various time and frequency domain features.

Accuracy among various frequency bands (theta, alpha, beta and gamma) has been compared using all 10 channels. Results show that higher frequency bands beta and gamma performs superior in emotion classification. Large quantity of Gamma activity is associated to peace and naturally happy feeling. 

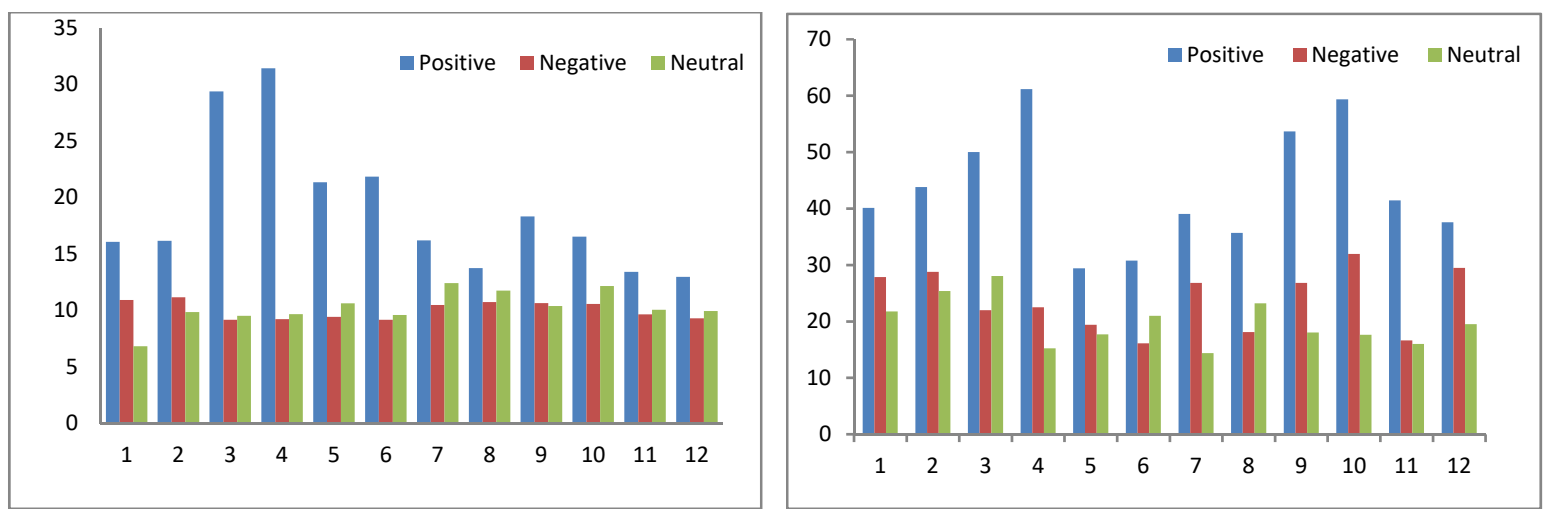

Fig.4. Energy performance of Gamma and Beta frequency band increases for positive emotion.

Previous research [Muhammad Adeel Asghar et.al.(2019), Mert et.al. (2018), Bhuvaneswari et.al. (2015)] says that, alpha band power is associated with relaxation, feeling of peaceful and positive effect. The Beta band power is associated with fast activity, attentive and busy mental activity accordingly beta power is more in Positive mind state.
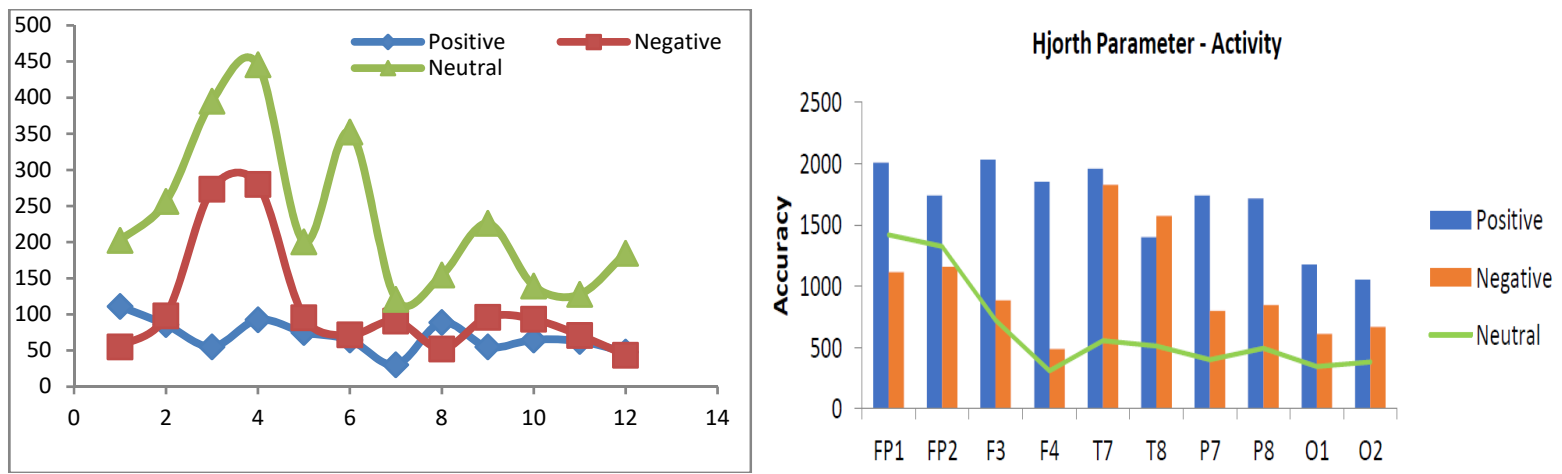

Fig. 5(a) Energy performance of Alpha frequency band increased in Neutral emotion. (b) Comparison of Hjorth parameter- Activity for various emotions

Left region of brain in involved in positive emotions and right region is related to negative emotions. So for this research we work on asymmetry of Hjorth Parameters and various frequency band features by considering FP1FP2, F3-F4, T7-F8, P7-P8 and O1-O2 electrode pairs.

Figure 6 presents classification accuracy of various regions of brain with FP1, FP2, F3, F4, T7, T8, P7, P8, O1 and $\mathrm{O} 2$ electrodes. Here KNN gives superior classification accuracy. Maximum classification accuracy for Left anterior part of brain is $60.19 \%$ and for right anterior part it is $57.61 \%$ using $\mathrm{KNN}$.

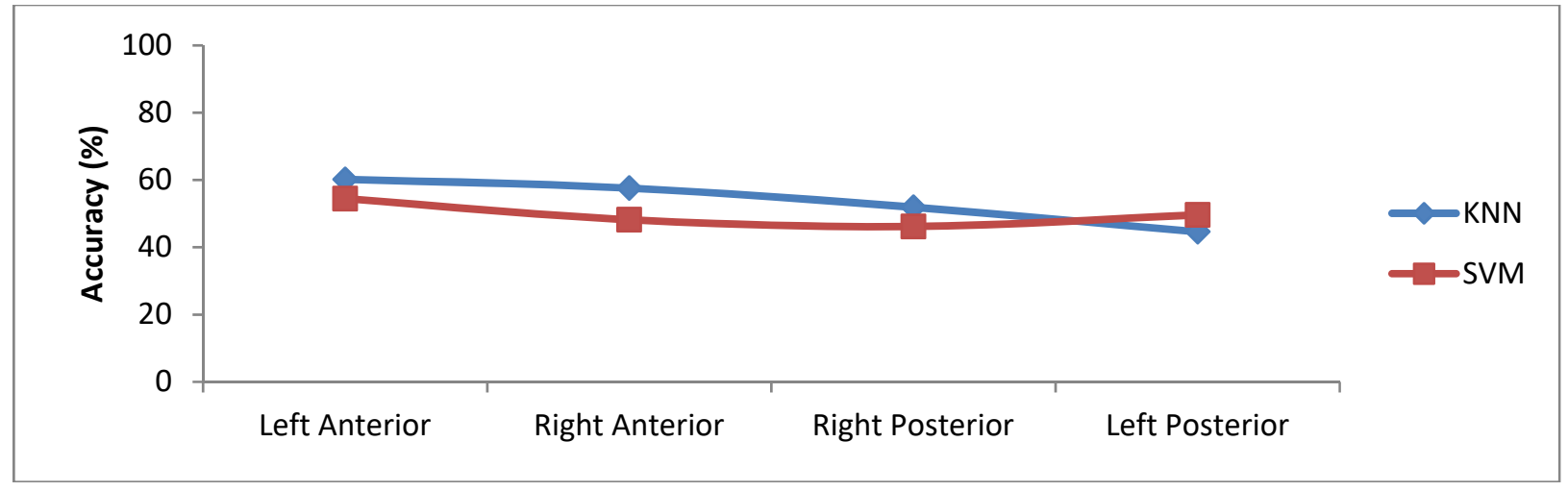

\section{Conclusions}

Fig. 6. Classification accuracy of various brain regions

The aim of this study was to classify various emotions like Positive, Negative and Neutral by fusion of various electrode pairs based on different brain regions. For this study, the publically available SEED database is used which consists of EEG data for three emotions and data was collected from 15 healthy subjects. 
For emotion classification DWT was used to extract features from EEG signal, while SVM and k-NN are used as classifiers. Left and right anterior region of the brain reveals good classification accuracy. For this work, we have considered various statistical features and various time frequency domain features of EEG signals. From results it became apparent that DWT features give good classification accuracy of $71.52 \%$. Also channel FP1 \& FP2 gives crest emotion classification accuracy. Also higher frequency bands like gamma and beta perform well for emotion categorization.

As shown from the results, feature selection and channel selection are keys to obtain good classification accuracy.

\section{References}

[1] Zangeneh S, Maghoolo K, Setarehdan S K, Motie Nasrabadi A,(2018), "Emotion classification through non linear EEG analysis using machine learning methods", Int. Clin. Neurosci J, 5, 135-149

[2] Faust O, Hagiwara Y, Hong T J, Lih O S, Acharya U R, (2018). "Deep learning for healthcare applications based on physiological signals :A review", Comput. Methods Progr. Biomed, 161,1-13

[3] Qi Xiong, Xinman Zhang, Wen-Feng Wang and Yuhong Gu,(2020), "A Parallel Algorithm Framework for Feature Extraction of EEG Signals on MPI , Hindawi ,Computational and Mathematical Methods in Medicine, Article ID 9812019

[4] Wei-Long Zheng, and Bao-Liang Lu, (2015),"Investigating Critical Frequency Bands and Channels for EEG-based Emotion Recognition with Deep Neural Networks, accepted by IEEE Transactions on Autonomous Mental Development (IEEE TAMD) 7(3): 162-175

[5] Danny Oud'e Bos, "EEG based emotion recognition, The influence of visual and auditory stimuli

[6] Xiang Li, Dawei Song, Peng Zhang, Yazhou Zhang, Yuexian Hou, Bin Hu,(2018), "Exploring EEG Features in Cross Subject Emotion Recognition", Frontiers in Neuroscience

[7] Kalyani Wagh, K Vasanth,(2019), "Electroencephalograph (EEG) Based Emotion Recognition System: A Review", Springer, Innovations in Electronics and Communication Engineering, Lecture Notes in Networks and Systems, Vol. 33

[8] Dan Nie, Xiao Wei Wang, Li Chen Shi, Bao Liang Lu, (2011), "EEG based emotion recognition during watching movies", 5th international IEEE EMBS conference on neural engineering

[9] Mu Li and Bao-Liang Lu, "Emotion Classification Based on Gamma-band EEG"

[10] Kalyani Wagh, K Vasanth,(2020), "Wavelet Transform Based Multichannel Emotion Detection through EEG Signal", Solid State Technology, Volume: 63, Issue: 5

[11] Muhammad Adeel Asghar, Mohammad Jamil Khan, Fawad, Yasar Amin, Muhammad Rizwan, Muhib Ur Rahman, Salman, Seyed Sajad Mirja (2019), "EEG Based Multi Modal Emotion Recognition Using Bag Of Deep Features: An Optimal Feature Selection Approach", Sensors

[12] Wei Liu, Jie Lin Qiu, Wei Long Zheng,(2019), "Multimodal Emotion Recognition Using Deep Conical Correlation Analysis, 2019

[13] Sadiq T, Yu X, Yuan Z, Fan Z, Rehman A. U., Li G, (2019), "Motor imagery EEG signals classification based on mode amplitude and frequency components using empirical wavelet transform", IEEE access , 7, 678-692

[14] Mert A, Akan A, (2018), "Emotion recognition from EEG channels by using multivariate empirical mode decomposition ", Pattern Anal. Appl., 21, 81-89

[15] Kevric J, Subasi A, (2017), "Comparison of signal decomposition methods in classification of EEG signals for motor imagery BCI system", Biomed Signal Process Control, 31, 398-406

[16] Gupta V, Chopda M D, Pachori R B, (2019),“ cross subject emotion recognition using flexible analytical wavelet transform from EEG signals", IEEE Sens J,19, 2266-2274

[17] Kalyani P. Wagh, K.Vasanth, Sagar B. Shinde(2022), "EEG Based Emotional State Recognition Using Time Frequency Parameters", Journal Harbin Institute of Technology, Vol 54 No 1, PP 101-109

[18] P. Bhuvaneswari, J Satheesh Kumar, (2015), "Influence of linear features in nonlinear EEG signals ", Science Direct Procedia computer science 47, pp -229-236

[19] Md.Asadur Rahman, Md.Foisal Hossain, Mazhar Hossain, Rasel Ahmmed,(2019), "Employing PCA and t-statistical approach for feature extraction and classification of emotion from multichannel EEG signal", Egyption Informatics Journal

[20] Duan R, Zhu J, Lu B, (2013), "Differential entropy feature for EEG based emotion classification", In proceedings of 6th International IEEE EMBS conference on neural engineering, San Diego,CA,USA,pp 81-84

[21] Henry Candra et.al., (2015), "Recognizing emotions from EEG sub bands using wavelet analysis",IEEE

[22] Seyyed Abed Hosseini, Mohammad Bagher Nagihi Sistani, (2011), "Emotion recognition method using entropy analysis of EEG signal",I.J.Image Graphics and Signal Processing,Vol.5, pp 30-36

[23] Zhang T, Zeng W, Cui Z, Zong Y, Li Y, (2019), "Spatial Temporal recurrent neural network for emotion recognition”, IEEE Trans.,49, 839-847

[24] Murugappn Murugappan,Nagarajan Ramchandran, Yaacob Sazali, (2010),“Classification of human emotion from EEG signal using discrete wavelet transform", J Biomedical science and engineering, Vol.3, pp 390-396

[25] Panagiotics C. Petrantonakis, Leontios J. Haddjileontiadis, (2011), "A novel emotion elicitation index using frontal brain asymmetry for enhanced EEG based emotion recognition" ,IEEE transactions on information technology in biomedicine, Vol. 15, No 5

[26] Li, PeiYang, Huan Liu, Yajinj,g Si, Cunbo Li, Fali Li, Xuyang Zhu, Xiaoye Huang et al. (2019). "EEG based emotion Recognition by combining functional connectivity network and local activations." IEEE Transactions on Biomedical Engineering

[27] Saadat Nasehi, Hossein Pourghassem, (2012), "An optimal EEG based emotion recognition algorithm using gabor features", WSEAS Transactions on signal processing,Vo. 8, Issue 3

[28] Kalyani Wagh, K Vasanth, (2019), " Review on Various Emotional Disorders by Analyzing Human Brain Signal Patterns (EEG Signals)", IEEE International Conference on Electrical, Computer and Communication Technologies

[29] Anagnostopoulos C N, IIiou T, Giannoukos I,(2015), "Features and classifiers for emotion recognition from speech : A survey from 2000 to 2011, Artificial Intelligence Rev. ,43,155-177

[30] Dan Nie, Xiao Wei Wang, Li Chen Shi, Bao Liang Lu, (2011), "EEG-Based Emotion Recognition Using Frequency Domain Features and Support Vector Machines", Springer-Verlag Berlin Heidelberg, ICONIP Part I, LNCS 7062, pp. 734-743

[31] You Yun Lee, Shulan Hsieh, (2014), "Classifying Different Emotional States By Means EEG Based Functional Connectivity Patterns" ,PLOS ONE, Vol.9, Issue 4

[32] Murugappn Murugappan, Rajamanickam Yuvaraj et.al., (2014),"On The Analysis Of EEG Power, Frequency And Asymmetry In Parkinson Disease During Emotion Processing", Behavioral And Brain Functions

[33] J.X.Chen, P.W.Zhang, Z.J. Mao, Y.F.Huang, D.M. Jiang, Y.N.Zhang, (2019), “Accurate EEG Based Emotion Recognition On Combined Features Using Deep Convolutional Neural Network", IEEE Access 
[34] Mohammad Soleymani, Sadjad Asghari Esfeden, Maja Pantic, Yun Fu, "Continuous emotion detection using EEG signals and facial expressions"

[35] Anne Claire Conneau, Slim Essid, (2014), “Assessment of new spectral features for EEG based emotion recognition”, IEEE International conference on acoustic, speech and signal processing

[36] Neep Hazarika, Jean Zhu Chen, Ah Chung Tsoi, Alex Sergejew, (1997), "Classification of EEG signals using the wavelet transform”, Signal Processing, pp 61-72

\section{Authors Profile}

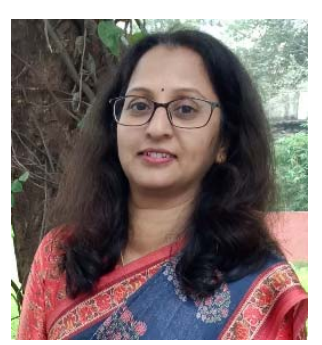

Ms. Kalyani P. Wagh working as Assistant Professor at JSPM Narhe Technical Campus Pune. She has completed his M.E. degree in Digital Systems from Pune University. She has 4 Conference papers and 3 Journal paper on her name. Her area of interest is Biomedical Signal Processing, Artificial intelligence and Machine learning.

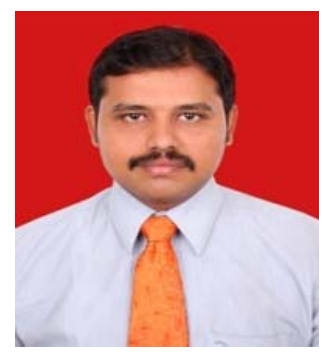

Dr. K. Vasanth was born in Madurai, Tamilnadu, India 0n $7^{\text {th }}$ July 1982 . He went on to complete his bachelor in Engineering in Electronics and communication from Madurai Kamaraj University. He scored $5^{\text {th }}$ rank in his Master of Engineering in Applied Electronics. He completed his Doctoral Degree in VLSI signal Processing. He played a key role as Project lead and System Engineer for the prestigious nano satellite sathyabamasat. He has published more than 65 research papers in renowned journals and conferences. The Major field of Interest is on Health care, Non Destructive testing of Metals and Non Linear filters.

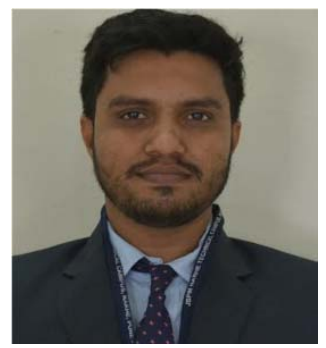

Dr. Sagar B. Shinde working as an Associate Professor \& Head of Department of Computer Science Engineering- Artificial Intelligence. He has completed his $\mathrm{PhD}$ in Electronics \& Communication Engineering and has a total $12+$ years of experience in teaching. He has 04 patents, 12 conference papers \& 36 journal papers on his name. His area of interest is image processing \& machine learning. He serves as a reviewer and editorial board member for various international journals $\&$ conferences. 